\title{
Brain magnetic resonance imaging findings in children with headache
}

\author{
Esra Gurkas ${ }^{a}$, M.D., Zeynep S. Karalok ${ }^{a}$, M.D., Birce D. Taskına, M.D., \\ Ummu Aydogmus ${ }^{a}$, M.D., Cahide Yilmaz , Assoc. Prof., Gulsah Bayram ${ }^{b}$, M.D.
}

\begin{abstract}
Introduction. The aim was to describe the findings on magnetic resonance imaging (MRI) in children with headache.

Population and methods. Retrospective review of the medical records of patients who were admitted to our pediatric outpatient neurology clinics with the complaint of headache between January 2013 and December 2014.
\end{abstract}

Results. A total of 478 patients (273 female, 205 male) were admitted with the complaint of headache. The types of headache were migraine in $218(45.6 \%)$, tension-type in 159 $(33.3 \%)$, secondary in $39(8.2 \%)$ and unspecified headaches in $62(13 \%)$ patients. Brain MRI was performed in $407(85 \%)$ patients and revealed cerebral abnormalities in $128(31.4 \%)$ patients; 5 patients had cerebral abnormalities relevant with headache, including tumors. Amongst the others 123 patients, the most common findings were 42 cases $(10 \%)$ of nonspecific white matter abnormalities, 17 cases $(4 \%)$ of enlarged perivascular spaces, 17 cases $(4 \%)$ of arachnoid cyst, 16 cases $(3.9 \%)$ of asymmetric ventricles, 12 cases $(2.9 \%)$ with Chiari type I and cerebellar tonsillar ectopia. Also, 17 (4.1\%) patients had extra-cerebral MRI abnormalities including sinusitis, mucosal thickening and retention cysts of sinuses.

a. Department of Pediatric Neurology, Ankara Pediatric\&Pediatric Hematology Oncology Training and Research Hospital, Ankara, Turkey.

b. Department of Radiology, Ankara Pediatric\&Pediatric Hematology Oncology Training and Research Hospital, Ankara, Turkey.

E-mail address:

Esra Gurkas, M.D.:

esragurkas@yahoo.com

Funding:

None.

Conflict of interest:

None.

Received: 2-4-2017

Accepted: 6-21-2017
Conclusions. In this study, the contribution of brain MRI in the diagnosis and management of the children with headache was still low.

Key words: headache, magnetic resonance imaging, child.

http: / / dx.doi.org/10.5546/ aap.2017.eng.e349

To cite: Gurkas E, Karalok ZS, Taskin BD, et al. Brain magnetic resonance imaging findings in children with headache. Arch Argent Pediatr 2017;115(6):e349-e355.

\section{INTRODUCTION}

Headache is a common disorder in children. The reported rates of headache prevalence during childhood range from $26.6 \%$ to 93.3\% ${ }^{1,2}$ In a study from Turkey, Karlı $\mathrm{N}$ et al. reported that the prevalence of recurrent headache in adolescents aged between 12 to 17 years was $52.2 \% .^{3}$
Most of the headaches in children are especially due to benign situations like primary headaches. The etiology of headache in children and adolescents can be predominantly determined with a detailed history and a comprehensive neurologic examination. Routine neuroimaging is not recommended in children with headache. The recommendations regarding imaging of children with headache were released by the Quality Standards Subcommittee of the American Academy of Neurology and the Practice Committee of the Child Neurology Society in 2002. According to these recommendations, indications for cranial imaging included acute severe headache, abnormal neurologic examination, a change in the pattern of headache, signs of increased intracranial pressure and coexistence of seizures. ${ }^{4}$ But in clinical practice, neuroimaging studies are commonly requested during the initial evaluation of children with headache due to the fear of missing an underlying serious disease and increasing parental demands. With the increasing rate of using neuroimaging procedures, detection of incidental abnormal findings which are unrelated to headache is also increasing.

In this study, we aimed to describe the findings on magnetic resonance imaging (MRI) in children with headache.

\section{POPULATION AND METHODS}

The study was a retrospective study conducted in a tertiary hospital at Ankara, Turkey. Children who were admitted to our pediatric outpatient neurology clinics with headache as the main presenting symptom between January 2013 and December 2014 were 
included. Patients with known intracranial lesion were excluded.

In our pediatric outpatient neurology clinic, a standardized approach for the patients with headache was used. All children were evaluated by a detailed history, systemic physical examination including arterial blood pressure measurement, detailed neurological and ophthalmologic examination. All data were saved as a structured file by pediatric neurologist at each visit. We retrospectively reviewed these data including age at presentation, sex, duration of illness, frequency of headache attacks, location, quality of headache, influence of physical activity, precipitating factor, associated neurologic symptoms and brain MRI findings. Patients were evaluated every three months after following the initial examination. The patients who were followed for at least 6 months were included in the study.

The types of headache were classified according to the third edition (beta version) of the International Classification of Headache Disorder. ${ }^{5}$ All headaches were mainly divided into two groups; primary or secondary headaches. Then, the primary headaches were divided into four groups: migraine, tension-type headache, trigeminal autonomic cephalalgias and other primary headaches. The criteria of primary headaches based on the specific features of the headache and associated symptoms. If the headache is missing one of the features required to fulfill all criteria for a subtype of headache and not fulfilling criteria for another headache disorder, the headache is classified in probable group, such as probable migraine or probable tension-type headache. In this study, the patients in probable groups were included in the definite diagnosis group. When the patient had a new headache that occurred for the first time in close temporal relation to another disorder that is known to cause headache, the patient was diagnosed with secondary headache. If a pre-existing primary headache became chronic in close temporal relation to such a causative disorder or a preexisting primary headache was made significantly worse in close temporal relation to a causative disorder such as sinusitis, the patient was included in the secondary group. If we couldn't classify the type of headache according to these criteria during the first evaluation, the headache was included as "unspecified" type.

The neuroimaging studies were performed according to the recommendations of the Quality Standards Subcommittee of the American Academy of Neurology and the Practice Committee of the Child Neurology Society in 2002. ${ }^{4}$ Additionally, the neuroimaging was also performed when the patients and/or the parents were so worried about an underlying serious brain disease and were not convinced by the physician.

The preferred choice of neuroimaging in patients with headache was MRI. All cerebral MRI studies were performed on a 1.5 Tesla MRI system (Infinion $1.5 \mathrm{~T}$, Philips, Holland). The same standardized protocol including T1weighted spin-echo images obtained from coronal, axial, and sagittal plane using a slime thickness of 4-7 mm, turbo spin-echo T2-weighted and fast fluid- attenuated inversion recovery-weighted images obtained from axial and coronal plane with a slice thickness of 5-7 $\mathrm{mm}$ and diffusion sequences were used. Other sequences such as application of contrast agent, magnetic resonance venography or magnetic resonance angiography were performed if clinically or radiologically indicated.

MRI results of the patients were firstly grouped as normal or abnormal. Secondly, abnormal results were classified as abnormalities relevant to headache and incidental findings.

Statistical analysis was performed using SPSS 17.0, Chicago, IL, USA. We used frequencies and cross-tables for data interpretation. For comparing groups, Pearson chi-square test, student $t$ test, one way ANOVA test and post hoc analysis were used. $\mathrm{P}<0.05$ was considered statistically significant.

The study was approved by the local ethic committee of the hospital.

\section{RESULTS}

A total of 478 patients ( 273 female, 205 male) were admitted with the complaint of headache between January 2013 and December 2014. Brain MRI was performed in $407(85 \%)$ patients and these patients were included in our study. The mean age at initial visit was $12.28 \pm 3.29$ years (2.6-18 years). The mean duration of headache before presentation was $14.09 \pm 11.15$ months. The types of headache were migraine in 218 $(45.6 \%)$, tension-type in $159(33.3 \%)$, secondary in $39(8.2 \%)$ and unspecified headaches in 62 $(13 \%)$ patients. There was no difference between the mean age of the patients with primary headache and the patients with secondary 
headache $(12.64 \pm 3.14$ years in patients with primary headache, $11.90 \pm 3.36$ years in patients secondary headache; $p=0.166$ ). Also there was no difference in sex distribution between primary and secondary headache group $(\mathrm{p}=0.47)$. Mean duration of headache before presentation was significantly lower in patients with secondary headache when compared with primary headache $(14.47 \pm 11.3$ months in patients with primary headache, $10.3 \pm 8.5$ months in patients with secondary headache; $\mathrm{p}=0.031$ ).

Overall, 407 (85\%) patients underwent brain MRI, which revealed cerebral abnormalities in $128(31.4 \%)$ patients. Abnormal brain MRI findings were divided into two categories as follows: 1) abnormalities relevant to headache such as tumors; 2) incidental abnormalities. Five patients had abnormalities relevant with headache. These patients were found to have space occupying lesion on MRI. They were evaluated by neurosurgeons and were operated. The first patient had frontally located low grade glial tumor (Figure 1-A), the second patient had primitive neuroectodermal tumor (Figure 1-B), the third one had medulloblastoma (Figure 1-C). The fourth patient had craniopharyngioma and the fifth patient also had a low grade glial tumor.

Amongst the other 123 patients, the most common incidental findings were 42 cases $(10 \%)$ of nonspecific white matter abnormalities, 17 cases $(4 \%)$ of enlarged perivascular spaces (Figure 2-A), 17 cases (4\%) of arachnoid cyst (Figure $2-B), 16$ cases $(3.9 \%)$ of asymmetric ventricles, 12 cases $(2.9 \%)$ with chiari type I (Figure 2-C) and cerebellar tonsillar ectopia. The other 19 patients had MRI abnormalities including $5(1 \%)$ cysterna magna enlargement (Figure 2-D), $3(0.7 \%)$ parsial empty sella, $3(0.7 \%)$ choroid plexus cyst and calcification, $1(0.2 \%)$ corpus callosum anomalies, $2(0.5 \%)$ pineal cyst, $1(0.2 \%)$ lipoma, $1(0.2 \%)$ vascular malformation, $1(0.2 \%)$ chronic infarct, $1(0.2 \%)$ leukodystrophy, $1(0.2 \%)$ hipocampal atrophy.

The headache in patients with Chiari type I and cerebellar tonsillar ectopia was generally temporally or frontally located, duration of headache was between 30 minutes to two hours, and not precipitated by cough or other Valsalvalike maneuvre so the feature of headache in these patients was not consisted with the criteria for the headache attributed to Chiari malformation type I. All these patients were evaluated by neurosurgeons and neurosurgical procedure was not required presently.

In patients with nonspecific white matter abnormalities, further investigations including cerebral magnetic resonance angiography, antinuclear antibody, anti-double-stranded DNA antibody and viral serology were performed to exclude the other potential etiologies of white matter disease. Clinical and radiological follow up were performed for these patients. No specific disease was detected so we considered that these white matter hyperintensities had no clinical importance.

The half of the patients with nonspecific white matter abnormalities had migraine but no difference between the frequency of nonspecific white matter abnormalities in migraine and the other headache types of patients was found $(\mathrm{p}=0.55)$.

Also, $17(4.1 \%)$ patients had extra-cerebral

FIGURE 1. Brain MRI of the patients with tumors, white arrows indicate lesion. (A) Axial T2-weighted image showing low grade glial tumor at the right frontal lobe $(B)$ Coronal T2-weighted image showing primitive neuroectodermal tumor at the right temporal lobe (C) Axial T2-weighted image showing medulloblastoma at cerebellum
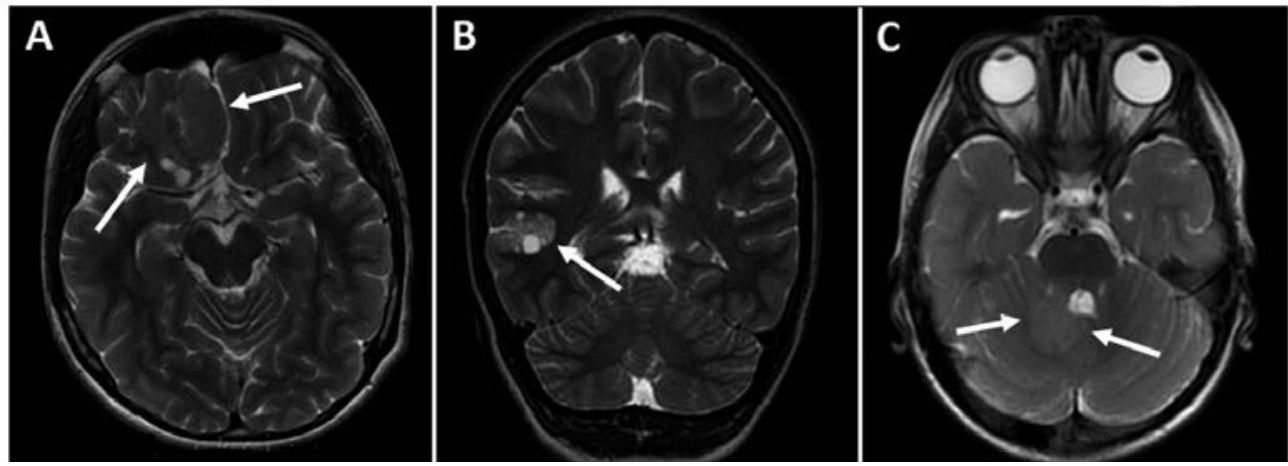
MRI abnormalities including sinusitis, mucosal thickening and retention cysts of sinuses. These patients were evaluated by otolaryngologist. The diagnosis of sinusitis in 13 patients was confirmed by clinical and nasal endoscopic examination. The headache in patients with sinusitis was exacerbated by pressure applied over the paranasal sinuses and significantly resolved in parallel with the resolution of the rhinosinusitis after treatment. The feature of the headache in patients with sinusitis meets the International Classification of Headache Disorder criteria for the headache attributed to acute rhinosinusitis.

\section{DISCUSSION}

Headache is a common disorder in pediatric age group. Patients with headache constitute the majority of the patients referred to pediatric neurology outpatient clinics. Migraine and tension-type headache are the common causes of headache in children..$^{6-9}$ Similar to the literature, migraine was the most common type of headache followed by tension type of headache in our series. The frequency of secondary headache was $8.2 \%$ in our study, also similar findings have been reported by previous studies. ${ }^{6-7}$
According to guidelines, performing a neuroimaging study on a routine basis is not recommended in children with headaches and with normal neurologic examination. However, in clinical practice, neuroimaging studies are commonly requested during the initial evaluation of children with headache due to the fear of missing an underlying serious disease and increasing parental demands. In previous studies especially from USA, the rates of neuroimaging studies for children with headache changes from $35.4 \%$ to $57.6 \% .^{10-12}$ In the present study, cerebral neuroimaging studies were performed in $85 \%$ of the patients. Rate of neuroimaging was higher in our study as the previous published studies from our country. ${ }^{7-9}$ The higher percentage may be due to the increased demands of the parents and also physicians because of the increase in malpractice lawsuits, busy practice conditions of outpatient clinics and therefore limited time to perform a detailed history and examination and also financial incentives of national health insurance funded by government.

Different abnormalities can be detected in brain MRI of patients with headache. These abnormalities may be relevant to headache and

FIGURE 2. Brain MRI of the patients, white arrows indicate lesion. (A) Axial T2-weighted image showing dilated perivascular spaces (B) Axial T2-weighted image showing arachnoid cyst located at the left anterior temporal region (C) Sagital T2weighted image showing chiari malformation (D) Axial T2-weighted image showing cysterna magna enlargement
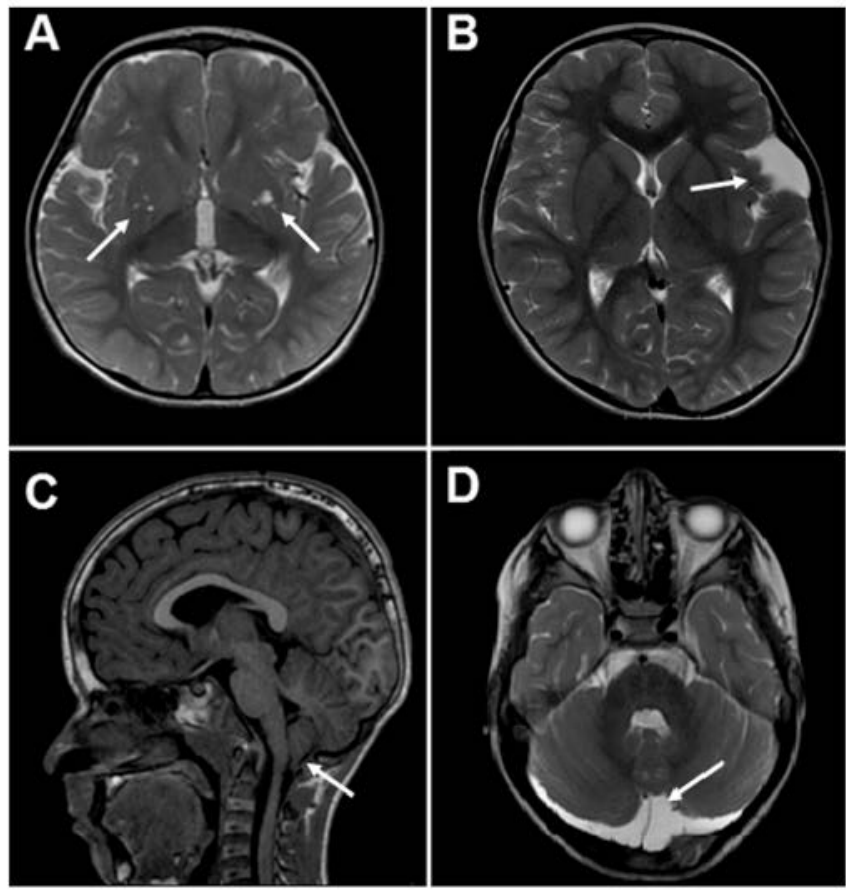
some of them may be incidentally detected lesions. The reported rates of abnormal neuroimaging findings in pediatric patients with headache has ranged from $9.3 \%$ to $21.6 \% .6,7,9,10,13$ With using modern sequences in children with headache, the rate of abnormal finding has raised to $52.8 \%$ but clinical gain of using these sequences was still limited. ${ }^{14}$ In our study, 35\% of patients had abnormal brain MRI findings.

Sinus disease was the major cause of headache in the patients with secondary headaches. In our study, sinus disease was found in 17 of children $(4.1 \%)$ who underwent imaging for headache. The frequency of sinus disease has previously been reported between $1.3 \%$ and $13.7 \%$ of patients imaged for headache. ${ }^{10,12,15}$ This is in line with our results.

Headache appears to be the presenting symptom in $17 \%$ to $41 \%$ of the patients with tumor but headache usually occurs with other symptoms such as vomiting, educational and behavioral problems, unsteadiness and visual difficulties. ${ }^{16-19}$ Approximately $10 \%$ of children with brain tumors, who have normal physical examination, present with headache as their only symptom. ${ }^{6}$ In a meta-analysis reviewing 16 studies including 19559 people without neurological or psychiatric symptoms, who underwent brain MRI as a research cases or controls or as recipients of commercial, clinical or occupational screening, only 135 people $(0.70 \%)$ were found to have neoplastic incidental brain findings. ${ }^{19}$ In our series, 5 children $(1.2 \%)$ underwent MRI for headache were found to have brain tumors. All these 5 children had normal neurologic and systemic examination.

Chiari type I malformation consists of protrusion of cerebellar tonsils $>5 \mathrm{~mm}$ below the foramen magnum. Cerebellar tonsil protrusion $<5 \mathrm{~mm}$ below the foramen magnum is defined as cerebellar tonsillar ectopia. ${ }^{20}$ The patients with Chiari type I malformations can present with headache, lower cranial nerve palsies, weakness and scoliosis. ${ }^{21}$ In our study, the headache pattern in patients with Chiari type I and cerebellar tonsillar ectopia was not found attributable to Chiari malformation. Chiari type I malformation and cerebellar tonsillar ectopia was found in 2.9\% of patients who underwent cranial MRI. Similarly, in previous studies in children with headache, Chiari type I malformation has been found in $2.7 \%$ to $5.8 \% .^{10,12,22}$ The prevalence of chiari type I malformation $(1.7 \%)$ and cerebellar tonsillar ectopia $(0.8 \%)$ was also $2.5 \%$ in pediatric patients attending to neurology clinics. ${ }^{20}$

Arachnoid cysts are cerebrospinal fluid contained sacs within the arachnoid membrane. The majority of arachnoid cysts are developmental but they may develop secondary to trauma, tumor or infection. Arachnoid cysts are mostly asymptomatic but they may produce symptoms occasionally because of compression and bleeding. ${ }^{23}$ In this study, arachnoid cyst present in $4 \%$ of patients. The prevalence of arachnoid cysts in previous headache studies is between $1 \%$ to $4.4 \% .^{10,12,13,24}$ In this study, imagings of the patients with arachnoid cysts showed only a mild mass effect, their headache symptoms were considered unrelated to the cyst.

Virchow-Robin spaces are projections of pia matter surrounding wall of the cerebral arteries. Virchow-Robin spaces are normal anatomical structures of central nervous system, usually smaller than $2 \mathrm{~mm}$ but sometimes can be dilated. In our series, dilated Virchow-Robin spaces were found in $4 \%$ of the patients imaged for headache. Similarly, Biedron A et al. evaluated 1348 MRI examinations of children patients who were hospitalized at pediatric neurology department and revealed the incidence of dilated VirchowRobin spaces as $3.93 \% .{ }^{25}$ Also in the study of Gupta et al. the incidence of enlarged perivascular spaces was $3.8 \% .^{20}$

Nonspecific white matter abnormalities are foci of ischemic demyelination and gliosis. ${ }^{26}$ These abnormalities are detected best as hyperintense foci on T2-weighted sequences. In this study, nonspecific white matter abnormalities were found in 42 of $127(10 \%)$ patients who underwent brain MRI. In previous studies, the rate of white matter abnormalities was ranged from $2.9 \%$ to $4.4 \% .^{7,8,10}$ Our result was higher than the rate in previously published studies. Also several studies in children and adults indicate that patients with migraine are at a higher risk of having white matter abnormalities on cranial MRI than patients without migraine. ${ }^{8,24,27}$ But the etiology of white matter abnormalities in migraine patients is not yet known. Also, in this study, we couldn't find any difference between the frequency of nonspecific white matter abnormalities in migraine and the other headache types of patients.

\section{CONCLUSION}

We have identified high rate of incidental abnormalities in children with headache. The majorities of these incidental abnormalities 
were not relevant with headache as well as not contribute to the treatment and the management of headache. Beside, these incidental abnormalities may also cause more concern in children and their parents. The contribution of brain MRI in the diagnosis and management of the children with headache was still low..

\section{REFERENCES}

1. Dooley JM, Gordon KE, Wood EP. Self-reported headache frequency in Canadian adolescents: Validation and followup. Headache 2005;45(2):127-31.

2. Rhee H. Prevalence and predictors of headaches in US adolescents. Headache 2000;40(7):528-38.

3. Karli N, Akiş N, Zarifoğlu M, et al. Headache prevalence in adolescents aged 12 to 17: a student-based epidemiological study in Bursa. Headache 2006;46(4):649-55.

4. Lewis DW, Ashwal S, Dahl G, et al. Practice parameter: evaluation of children and adolescents with recurrent headaches: report of the Quality Standards Subcommittee of the American Academy of Neurology and the Practice Committee of the Child Neurology Society. Neurology 2002;59(4):490-8.

5. Headache Classification Committee of the International Headache Society (IHS). The International Classification of Headache Disorders. 3rd ed (beta version). Cephalalgia 2013;33(9):629-808.

6. Rho YI, Chung HJ, Suh ES, et al. The role of neuroimaging in children and adolescents with recurrent headaches-multicenter study. Headache 2011;51(3):403-8.

7. Yılmaz Ü, Çeleğen M, Yılmaz TS, tal. Childhood headaches and brain magnetic resonance imaging findings. Eur J Paediatr Neurol 2014;18(2):163-70.

8. Bayram E, Topcu Y, Karaoglu P, et al. Incidental white matter lesions in children presenting with headache. Headache 2013;53(6):970-6.

9. Alehan FK. Value of neuroimaging in the evaluation of neurologically normal children with recurrent headache. J Child Neurol 2002;17(11):807-9.

10. Schwedt TJ, Guo Y, Rothner AD. "Benign" imaging abnormalities in children and adolescents with headache. Headache 2006;46(3):387-98.

11. Graf WD, Kayyali HR, Alexander JJ, et al. Neuroimaginguse trends in nonacute pediatric headache before and after clinical practice parameters. Pediatrics 2008;122(5):e1001-5.

12. Lewis DW, Dorbad D. The utility of neuroimaging in the evaluation of children with migraine or chronic daily headache who have normal neurological examinations. Headache 2000;40(8):629-32.

13. Wöber-Bingöl C, Wöber C, Prayer D, et al. Magnetic resonance imaging for recurrent headache in childhood and adolescence. Headache 1996;36(2):83-90.

14. StreibertPF, Piroth W, MansourM, et al. Magnetic resonance imaging of the brain in children with headache: the clinical relevance with modern acquisition techniques. Clin Pediatr (Phila) 2011;50(12):1134-9.

15. Maytal J, Bienkowski RS, Patel M, et al. The value of brain imaging in children with headaches. Pediatrics 1995;96(3 Pt 1):413-6.

16. Wilne SH, Ferris RC, Nathwani A, et al. The presenting features of brain tumours: a review of 200 cases. Arch Dis Child 2006;91(6):502-6.

17. Hayashi N, Kidokoro H, Miyajima Y, et al. How do the clinical features of brain tumours in childhood progress before diagnosis? Brain Dev 2010;32(8):636-41.

18. Nelson S, Taylor LP. Headaches in brain tumor patients: primary or secondary? Headache 2014;54(4):776-85.

19. Morris Z, Whiteley WN, Longstreth WT Jr, et al. Incidental findings on brain magnetic resonance imaging: systematic review and meta-analysis. BMJ 2009;339:b3016.

20. Gupta SN, Belay B. Intracranial incidental findings on brain MR images in a pediatric neurology practice: a retrospective study. J Neurol Sci 2008;264(1-2):34-7.

21. Greenlee JD, Donovan KA, Hasan DM, et al. Chiari I malformation in the very young child: the spectrum of presentations and experience in 31 children under age 6 years. Pediatrics 2002;110(6):1212-9.

22. Medina LS, Pinter JD, Zurakowski D, et al. Children with headache: clinical predictors of surgical spaceoccupying lesions and the role of neuroimaging. Radiology 1997;202(3):819-24.

23. Gosalakkal JA. Intracranial arachnoid cysts in children: a review of pathogenesis, clinical features, and management. Pediatr Neurol 2002;26(2):93-8.

25. Eidlitz-Markus T,Zeharia A, Cohen $\mathrm{YH}$, etal.Characteristics and management of arachnoid cyst in the pediatricheadache clinic setting. Headache 2014;54(10):1583-90.

26. Biedroń A, Steczkowska M, Kubik A, et al. Dilatation of Virchow-Robin spaces in children hospitalized at pediatric neurology department. Neurol Neurochir Pol 2014;48(1): 39-44.

27. Candee MS, McCandless RT, Moore KR, et al. White matter lesions in children and adolescents with migraine. Pediatr Neurol 2013;49(6):393-6.

28. Swartz RH, Kern RZ. Migraine is associated with magnetic resonance imaging white matter abnormalities: a metaanalysis. Arch Neurol 2004;61(9):1366-8. 